\title{
Brain Dynamics in Spider-Phobic Individuals Exposed to Phobia-Relevant and Other Emotional Stimuli
}

\author{
Jaroslaw M. Michalowski, Christiane A. Melzig, \\ and Almut I. Weike \\ University of Greifswald
}

Jessica Stockburger and Harald T. Schupp

University of Konstanz

\author{
Alfons O. Hamm \\ University of Greifswald
}

\begin{abstract}
Dense sensor event-related brain potentials were measured in participants with spider phobia and nonfearful controls during viewing of phobia-relevant spider and standard emotional (pleasant, unpleasant, neutral) pictures. Irrespective of the picture content, spider phobia participants responded with larger P1 amplitudes than controls, suggesting increased vigilance in this group. Furthermore, spider phobia participants showed a significantly enlarged early posterior negativity (EPN) and late positive potential (LPP) during the encoding of phobia-relevant pictures compared to nonfearful controls. No group differences were observed for standard emotional materials indicating that these effects were specific to phobia-relevant material. Within group comparisons of the spider phobia group, though, revealed comparable EPN and LPP evoked by spider pictures and emotional (unpleasant and pleasant) picture contents. These results demonstrate a temporal unfolding in perceptual processing from unspecific vigilance (P1) to preferential responding (EPN and LPP) to phobia-relevant materials in the spider phobia group. However, at the level of early stimulus processing, these effects of increased attention seem to be related to emotional relevance of the stimulus cues rather than reflecting a fear-specific response.
\end{abstract}

Keywords: fear, emotion, attention, phobia, event-related potentials (ERPs)

Supplemental materials: http://dx.doi.org/10.1378/a0015550.supp

Phobic fear is a response to explicit threat, which is rooted in the defensive system of the mammalian brain (Lang, Davis, \& Öhman, 2000; Öhman \& Mineka, 2001). Once activated, this system initiates a cascade of behavioral adjustments including increases in autonomic arousal (Fredrikson, 1981; Hare, 1973; LeDoux, 1987; Prigatano \& Johnson, 1974) and, depending upon the distance of the phobic object, freezing or escape to cope with the threat (Fanselow, 1994). Moreover, the human startle response, a defensive reflex serving a protective function, is facilitated when individuals with specific phobia (e.g., small animal and blood phobia) are confronted with pictures of their phobic objects (Globisch, Hamm, Esteves, \& Öhman, 1999; Hamm, Cuthbert, Globisch, \& Vaitl, 1997). The startle probe methodology allows assessing the speed of defensive activa-

Jaroslaw M. Michalowski, Christiane A. Melzig, Almut I. Weike, and Alfons O. Hamm, Department of Biological and Clinical Psychology, University of Greifswald; Jessica Stockburger and Harald T. Schupp, Department of Psychology, University of Konstanz.

Jaroslaw M. Michalowski is now at the Institute of Psychology, University of Gdansk.

This study was supported by a grant from the German Academic Exchange Service (DAAD), and by a grant from the Federal Ministry of Education and Research to Alfons Hamm.

Correspondence concerning this article should be addressed to Jaroslaw M. Michalowski, Department of Biological and Clinical Psychology, University of Greifswald, Franz-Mehring-Strasse 47/48, 17487 Greifswald, Germany. E-mail: psyjmi@univ.gda.pl tion. Potentiated startle reflex during viewing of phobia-related stimuli was observed already $300 \mathrm{~ms}$ after the onset of the phobic stimulus demonstrating the rapid engagement of the fear system (Globisch et al., 1999). However, in addition to engaging somatic, autonomic, humoral, and motor responses, fear cues are held to capture selective attention already modulating processes of stimulus perception and evaluation (Öhman, Flynkt, \& Esteves, 2001). Measuring event-related brain potentials, the current study aimed to detail the preferential processing of phobia-specific cues at the level of specific processing stages with regard to the attention capture of nonphobic emotional cues (cf., Hamm, Schupp, \& Weike, 2003).

A number of functional neuroimaging studies revealed increased perceptual processing of phobia-related stimuli in patients with specific phobia. Fredrikson and colleagues were among the first to observe the increased activation of secondary visual areas to feared materials in participants with snake phobia (Fredrikson et al., 1993). These findings have been confirmed in several recent studies showing increased activations in the lateral occipital, posterior parietal, and inferior temporal cortex in small animal phobic volunteers during processing of pictures of spiders or snakes relative to neutral materials (Dilger et al., 2003; Fredrikson, Wik, Annas, Ericson, \& Stone-Erlander, 1995; Paquette et al., 2003; Sabatinelli, Bradley, Fitzsimmons, \& Lang, 2005). Consistent with the notion that facilitated perceptual stimulus processing may be regulated by limbic structures, individuals with small animal phobia also exhibited increased amygdala and insula activations when 
confronted with pictures of their feared cues (Carlsson et al., 2004; Dilger et al., 2003; Fredrikson \& Furmark, 2003; Sabatinelli et al., 2005; Schienle, Schäfer, Walter, Stark, \& Vaitl, 2005; Straube, Mentzel, \& Miltner, 2006; Wendt, Lotze, Weike, Hosten, \& Hamm, 2008).

Recent studies have begun to explore the temporal dynamics of attentional modulation of stimulus processing relying on the measurement of event-related brain potentials. Most consistent findings emerged regarding the late positive potential component, which is thought to index attention processes related to conscious stimulus recognition and elaborate processing of motivationally significant stimuli (Del Cul, Baillet, \& Dehaene, 2007; Nieweunhuis, Aston-Jones, \& Cohen, 2005; Schupp, Flaisch, Stockburger, \& Junghöfer, 2006; Sergent, Baillet, \& Dehaene, 2005). Exploring the processing of fear-related, low-arousing pleasant, and neutral pictures in small animal phobic and healthy volunteers, Miltner and colleagues reported increased amplitudes of the late positive potential (LPP) to feared cues in individuals with animal phobia (Miltner et al., 2005). Studying spider and snake phobics, similar results were reported in a study assessing fearful picture processing in the context of a probabilistic classification task (Kopp \& Altmann, 2005). Furthermore, spider phobics showed enhanced LPP amplitudes to feared cues when the picture materials (spiders, flowers, and birds) were either task relevant or task irrelevant, that is, identification and emotional stroop task, respectively (Kolassa et al., 2005). Taken together, across studies, pictures of fearful materials elicited enhanced LPP amplitudes in small animal phobics revealing the increased attention capture processing of fearrelevant stimulus materials.

Building upon these findings, the present study aimed to advance the understanding of enhanced LPP amplitudes to fearrelevant cues in participants with spider phobia. Previous studies showed that the LPP enhancement is fear specific in that snake and spider phobics have selectively enhanced LPP amplitudes to fearrelevant stimulus materials. Although these data demonstrate the plasticity in fear cue processing at the level of stimulus perception, it is currently unclear how these findings relate to the processing of fear-irrelevant but also high-arousing aversive and appetitive stimuli. With regard to the startle reflex, it was shown that the startle reflex was potentiated to fear-relevant compared to nonfeared highly arousing aversive stimuli (Hamm et al., 1997). Thus, at the level of response output measures, enhanced defensive responding to feared stimulus materials is specifically augmented to feared materials. However, contrasting findings for perceptual processes are suggested by two recent fMRI studies reporting that the enhanced activation in the amygdala and visual-associative regions to fear-relevant cues was not enlarged beyond the activation for nonfeared high-arousing emotional stimuli (Sabatinelli et al., 2005; Wendt et al., 2008). Studying this issue seems important considering the critical role of attention processes in current etiological models of fear and anxiety (e.g., Mathews \& Mackintosh, 1998; Mogg \& Bradley, 1998). Specifically, the hypothesis that attentional bias to threat might be the core problem in anxiety disorders (Williams, Watts, MacLeod, \& Mathews, 1997) would receive strong support if, similar to the startle reflex, the LPP amplitude is augmented for fear-relevant cues compared to other emotionally arousing stimulus materials.

Although less consistent, previous studies also found a modulation of event-related potential (ERP) components preceding the
LPP component. For instance, a previous study observed differences of the P1 component in spider phobics compared to healthy volunteers. Examining the processing of schematic spider and flower stimuli, the P1 was enhanced in spider phobics over occipital regions. However, this effect was not specific to the picture materials, that is, observed for spider and flower pictures, and was similarly observed in a social phobia group (Kolassa, Musial, Kolassa, \& Miltner, 2006). The P1 has been linked to visuospatial attention and is thought to reflect a gain mechanism enhancing processing in extrastriate visual cortex (Mangun, Hillyard \& Luck, 1993). For instance, enhanced $P 1$ has been observed when target stimuli were presented in attended compared to unattended spatial locations or when target stimuli were preceded by fearful rather than neutral faces (Clark \& Hillyard, 1996; Pourtois, Grandjean, Sander, \& Vuilleumier, 2004). Accordingly, enhanced P1 waves may reflect a stage of unspecific hypervigilance and facilitated visual processing of any stimuli. Based on these findings, the present study determined whether spider phobics are hypervigilant toward any stimulus materials as indexed by the P1 considering not only fearful and neutral stimulus materials but also pleasant and unpleasant pictures depicting natural scenes (rather than schematic drawings).

Subsequent to the P1, there is initial evidence that visual processing in small animal phobics is facilitated in a later time window from 175 to 275 ms. Specifically, Kopp and Altmann (2005) observed an increased posterior negativity to feared materials in individuals with snake and spider phobia. Similar effects have been consistently reported in studies examining emotional picture processing. Specifically, pleasant and unpleasant picture processing is associated with an early posterior negativity relative to neutral pictures (cf. Schupp et al., 2006). Furthermore, a similar brain signature was observed in explicit attention studies. Attention to distinct stimulus features (e.g., color or form) or higherorder categorizations defined by semantic meaning is associated with a posterior negativity occurring 150 to $350 \mathrm{~ms}$ after stimulus onset. This early posterior negativity effect is thought to reflect a transitory processing period increasing the allocation of processing resources in later processing stages. These findings suggest that the fear-specific modulation of the LPP is already present at an earlier, fleeting processing stage suggesting a considerable earlier time of fear-selective stimulus processing. The current study provided a conceptual replication of the finding that the EPN is specifically enhanced to fear-relevant pictures in spider phobics and allowed additional explorations in relation to high-arousing emotional picture materials.

The main aim of the present study was to determine the time course of fearful stimulus processing in spider phobics compared to healthy volunteers focusing on the P1, EPN, and LPP components, respectively. Accordingly, dense sensor ERPs were measured while spider phobic and nonphobic participants passively viewed phobia-relevant pictures as well as unpleasant, pleasant, and neutral stimuli from the International Affective Picture System (Lang, Bradley, \& Cuthbert, 2005). Based on a previous study (Kolassa et al., 2006), it was predicted that the P1 component is enlarged in spider phobics compared to healthy volunteers irrespective of the hedonic valence of the stimulus materials. Subsequently, indexed by the EPN and LPP components (cf., Kopp \& Altmann, 2005; Kolassa et al., 2005; Miltner et al., 2005), a fear-specific modulation was expected contrasting the processing of fearful stimuli in spider-fearful and healthy groups. Moreover, 
presenting other emotional arousing pictures allowed a more refined assessment of fear-specific processing in spider phobics. Specifically, it was determined whether the EPN or LPP is increased in amplitude when phobics view their fear-related cues compared to high-arousing emotional picture contents. Confirmation of this hypothesis would support the hypothesis of a selective attention bias toward fear cues at distinct processing stages implicated in stimulus selection (EPN) and conscious stimulus regulation and elaborate processing (LPP; Del Cul et al., 2006; Schupp et al., 2006; Sergent et al., 2005). Finally, with regard to standard emotional materials, increased EPN and LPP amplitudes were expected for the comparison of emotional (pleasant and unpleasant) and neutral picture materials (Schupp et al., 2006).

\section{Method}

\section{Participants}

A total of 43 participants were selected from a questionnaire screening of 336 students at the University of Greifswald. At time of recruitment, all participants completed the German version of the 31-item spider phobia questionnaire (SPQ; German version, Hamm, 2006). Twenty participants (15 females) were selected after reporting elevated spider fear on the SPQ and were included in the spider phobia group if their scores were $\geq 16$ or $\geq 13$ for females or males respectively, thus scoring above the 85 th percentile of the distribution $(M=20.3, S D=2.9$ for females and $M=$ $17.8, S D=5.7$ for males). This uneven gender distribution $(75 \%$ females) corresponds with epidemiological findings showing unequivocally that $70 \%$ to $80 \%$ of all patients diagnosed with specific phobia (animal type) are females (Curtis et al., 1998). The remaining 23 participants (15 females) of the control group reported lower levels of spider fear, that is, scoring below the 67th percentile of the distribution $(M=2.9, S D=2.8$ for females and $M=3.5, S D=2.7$ for males). ${ }^{1}$

\section{Experimental Stimuli and Procedure}

Participants viewed 144 color pictures classified into four categories: 36 pleasant (moderately arousing [e.g., cute animals, babies, and family scenes] and highly arousing [e.g., erotic couples]), 36 neutral (e.g., landscapes, buildings, and neutral people), 36 unpleasant (moderately arousing [e.g., human and animal attack] and highly arousing [e.g., mutilation]) selected from the International Affective Picture System (IAPS; Lang et al., 2005), and 36 phobia-relevant spider pictures originated from our own picture pool (see Hamm et al., 1997; Globisch et al., 1999; Wendt et al., 2008) successfully used in previous studies.

Participants were seated in a recliner in a dimly lit and soundattenuated room and viewed the stimuli displayed on the 20 " computer monitor located approximately $1.5 \mathrm{~m}$ from their eyes $\left(11^{\circ}\right.$ of visual angle). Each picture was presented for $1,500 \mathrm{~ms}$, preceded by a fixation cross $(500 \mathrm{~ms})$ and followed by an intertrial interval of 750 , 1,000 , or $1,250 \mathrm{~ms}$ (in random order). Each picture was displayed twice in three different orders that were counterbalanced across participants. Consecutive presentation of two stimuli from the same category was avoided. At the end of the session the EEG sensors were removed and each participant was asked to view each picture once more as long as desired and to press a button to terminate picture presentation. After the slide offset the participant evaluated his or her subjective experience of valence and arousal on a computerized version of the Self-Assessment Manikin (Bradley \& Lang, 1994).

\section{Apparatus}

Electrophysiological data were collected from the scalp using a 129-channel system (Electrical Geodesics, Inc., Eugene, OR). Scalp impedance was kept below $30 \mathrm{k} \Omega$ for each sensor as recommended by the EGI-systems guidelines. EEG data were continuously recorded in the 0.1 - to $100-\mathrm{Hz}$ frequency range with a sampling rate of $250 \mathrm{~Hz}$. The vertex sensor served as a reference electrode. Continuous EEG data were low pass filtered at $40 \mathrm{~Hz}$ using digital filtering before stimulus synchronized epochs were extracted from $120 \mathrm{~ms}$ before to $1,000 \mathrm{~ms}$ after picture onset. The raw EEG epochs were passed through a computerized artifact detection algorithm (Junghöfer, Elbert, Tucker, \& Rockstroh, 2000). Artifact rejection was based upon boundary values of three parameters: maximal absolute value over time, standard deviation over time, and maximal temporal gradient over time. In the first step, the data with common reference were used to detect contaminated sensors and sensor epochs. Based on artifact-free data, averaged reference was calculated. The data transformed to averaged reference were used to detect global artifacts and reject contaminated trials. Overall, approximately $30 \%$ of the trials were rejected because of artifacts. These rejected trials were equally distributed across all stimulus categories. For the remaining trials, rejected single channels were estimated by a spherical spline interpolation based on all remaining sensors on a trial-by-trial base.

\section{Data Analysis}

Following prior research with this paradigm (Schupp, Junghöfer, Weike, \& Hamm, 2003), visual inspection of the ERP waveforms as well as single-sensor waveform analyses were used in concert to identify the temporal and spatial characteristics of the ERP modulation as a function of picture category, group, and laterality.

To replicate previous findings, single-sensor waveform analyses were carried out by calculating repeated measures ANOVAs including the within-factors Picture Category (pleasant vs. neutral vs. unpleasant) and the between-factor Group (spider phobics vs. controls) for each time point and each individual sensor. To avoid false positives and to assure a more stringent alpha-level adjustment, significant effects were only considered meaningful when observed for at least eight continuous data points $(32 \mathrm{~ms})$ and two neighboring sensors. Inspection of the ERP waveforms revealed the presence of early (EPN) and late (LPP) preferential affective processing for sensor clusters and time intervals reported in previous studies (Schupp et al., 2003).

Moreover, visual inspection as well as single-sensor waveforms analyses revealed differences in the ERP waveforms to phobiarelevant pictures between the phobia group and nonfearful control participants in the time window of the P1, the EPN and the LPP. For detailed analyses of these effects, mean amplitudes averaged within the time windows and sensor clusters identified by both visual inspection and single-sensor waveform analyses were in-

\footnotetext{
${ }^{1}$ The gender distribution in the control group was kept comparable to the phobia group to control for possible gender effects on the brain potentials.
} 
cluded in further statistical analyses. The $P l$ was further analyzed in the time window from 80 to $132 \mathrm{~ms}$ after picture onset in two posterior sensor clusters comprising the following sensors: 70, 71, $72,74,75$ in the left hemisphere and 77, 83, 84, 89, 90 in the right hemisphere. The EPN was analyzed within the time window from 240 to $292 \mathrm{~ms}$ after picture onset in posterior-temporal sensor clusters comprising the following sensors: $47,50,51,56,57,58$ in the left hemisphere and 97, 98, 101, 102, 103, 108 in the right hemisphere. The statistical analyses of the $L P P$ were calculated in the time window from 428 to $588 \mathrm{~ms}$ after picture onset in two centro-parietal sensor clusters: $7,31,32,38,43,53,54$ in the left hemisphere, and 80, 81, 87, 88, 94, 106, 107 in the right hemisphere (see Figure 1). Separate between group analyses were carried out for the phobia-relevant picture category by calculating repeated measures ANOVAs including Laterality (right vs. left) as within factor and Group (spider phobics vs. controls) as a between factor. To determine the specificity of group differences, ERP components elicited by standard affective materials were submitted to ANOVAs including Picture Category (neutral vs. unpleasant vs. pleasant), Laterality, and Group.

A further analysis served to examine whether phobia-relevant picture processing is enhanced in the spider phobia group when contrasted with the processing of emotional (pleasant and unpleasant) picture contents. Accordingly, the EPN and LPP elicited by phobia-relevant pictures were further compared to those evoked by the standard affective materials using repeated measures ANOVAs including Picture Category (phobia-relevant vs. neutral vs. unpleasant vs. pleasant) and Laterality (right vs. left) as within subjects factors and Group (spider phobics vs. controls) as a between factor. Planned post hoc contrasts compared spider pictures with pleasant and unpleasant picture contents separately for both groups.

\section{Results}

\section{Valence and Arousal Ratings}

The valence and arousal ratings of this sample corresponded with the standard affective ratings on which the picture selection was based. As expected, participants with spider phobia rated pictures of spiders as being significantly more unpleasant, $t(41)=$ $8.31, p<.001$, and arousing, $t(41)=7.11, p<.001$, than controls.

\section{Event-Related Potential Between Group Comparisons: Spider Phobia Versus Control Group}

P1. Figure 2 illustrates the overall grand average ERPs elicited in spider phobia participants and controls over a representative left occipital sensor (\#71; top panel) during the processing of the four picture categories (phobia relevant, neutral, pleasant, and unpleasant) and corresponding scalp potential difference maps (spider phobia minus control group) for these stimulus materials (bottom panel). The statistical analysis revealed that spider pictures elicited a significantly larger P1 in spider phobics than in controls at occipital sensors, Group, $F(1,41)=6.55, p<.05$. However, participants with spider phobia not only responded with a larger P1 to their phobia-relevant stimuli but also showed a similar P1 increase during viewing of phobia-irrelevant unpleasant, pleasant, and even neutral pictures, Group, $F(1,41)=4.34, p<.05$. This increased P1 wave in the spider phobia group to all visual stimuli was similarly pronounced over the right and left hemisphere, Group $\times$ Laterality, $F(1,41)=1.23, n s .^{2}$

$E P N$. Figure 3 illustrates the ERP waveforms for a representative right temporo-occipital sensor (\#98; top panel) and corresponding scalp potential difference maps (bottom panel) for spider pictures and the three standard picture categories as a function of group. The statistical analysis of the ERP waveforms elicited by spider pictures at the posterior-temporal sensor cluster in the EPN time window resulted in a significant Group $\times$ Laterality interaction, $F(1,41)=4.82, p<.05$. Follow-up comparisons performed for the right hemisphere showed that spider pictures evoked a more negative ERP shift in the spider phobia group than in controls, $t(41)=2.26, p<.05$. This significant group difference was not observed for the left hemisphere, $t(41)<1$, ns. No group differences between participants with spider phobia and controls were observed during the encoding of the other emotional (unpleasant and pleasant) and neutral stimulus materials, Group, $F(1,41)<1$, $n s ;$ Group $\times$ Laterality, $F(1,41)=2.09$, ns.

$L P P$. As illustrated in Figure 4 for a representative right centro-parietal sensor (\#88; top left) spider pictures evoked significantly larger LPPs in spider phobics than in controls, $F(1,41)=$ $4.14, p<.05$. This effect did not differ significantly as a function of Laterality, $F(1,41)<1$, ns. As illustrated in Figure 4 (right) there were no significant group differences for standard affective materials, Group, $F(1,41)<1$, ns; Group $\times$ Laterality, $F(1$, $41)=1.37, n s$.

\section{Event-Related Potential Within Group Comparisons: Phobia-Relevant Versus Other Emotional Stimuli}

EPN. The statistical analyses of the ERP waveforms elicited by phobia-relevant and standard affective pictures at the posteriortemporal sensor clusters in the EPN time window revealed a significant group effect for the right, $F(3,123)=4.31 ; p<.05$, and no group difference for the left hemisphere, $F(3,123)<1$, ns. Follow-up tests calculated for the right posterior-temporal sensor cluster revealed that spider pictures elicited a significantly larger EPN than neutral pictures but only in the group of spider phobia participants, Category $\times$ Group, $F(1,41)=6.0, p<.05$. Moreover, replicating previous findings, pleasant and unpleasant pictures also elicited a significantly larger EPN than neutral pictures, $F(2,82)=42.39, p<.001$, a pattern that was comparable in both groups, Category $\times$ Group, $F(2,82)=2.23$, $n s$. Finally, comparing the EPN to spider pictures with those during processing of other emotional (pleasant and unpleasant) pictures revealed, that the EPN to spider pictures did not differ from the EPN amplitudes evoked by the other emotional unpleasant and pleasant pictures in spider phobia participants, $t \mathrm{~s}(19)<1.4, n s$, but were significantly

\footnotetext{
${ }^{2}$ Corresponding group differences were observed in a similar time window over centro-parietal brain areas. The 2 (Laterality) $\times 2$ (Group) analysis of variance performed for spider pictures resulted in a significant main effect of Group, $F(1,41)=5.92, p<.05$, indicating that these contents elicited more negative potentials in spider phobics than in controls. The same trend was observed for standard affective contents, Group, $F(1,41)=3.35, p=.079$, that was, however, more pronounced for the right relative to the left hemisphere, Laterality $\times$ Group, $F(1,41)=4.75$, $p<.01$.
} 

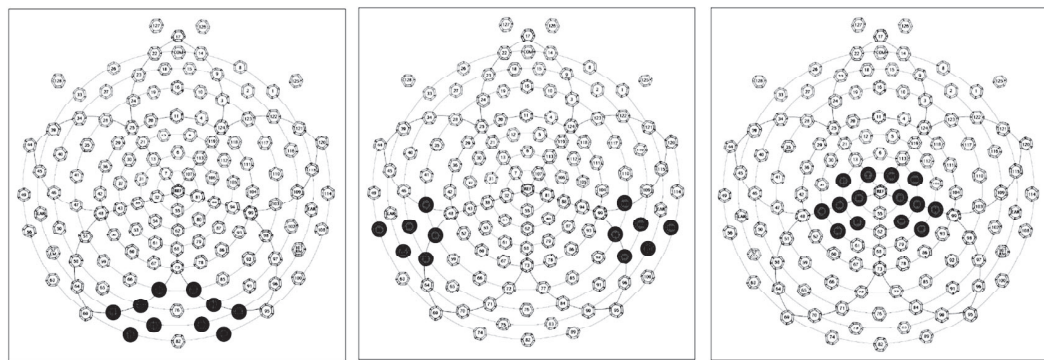

Figure 1. Sensor outline of the 129-Geodesic sensor net (image courtesy of Electrical Geodesics, Inc.) with colored sensor clusters used to quantify the P1 as well as the early posterior negativity and the late positive potentials

smaller relative to the EPN amplitudes elicited by the other emotional pictures in the control group, $t \mathrm{~s}(22)>2.9, p \mathrm{~s}<.01$.

$L P P$. Figure 5 depicts the mean LPP amplitudes of the centroparietal sensor cluster elicited during viewing of pleasant, neutral, unpleasant, and spider pictures in participants with spider phobia (left panel) and controls (right panel). Spider pictures elicited significantly larger LPP amplitudes than neutral materials, $F(1$, $41)=107.60, p<.001$, and this effect was significantly stronger for the spider phobia group than for the controls, Category $\times$ Group, $F(1,41)=8.10, p<.01$, in support of the results from the between group comparisons. Participants with spider phobia did not respond with larger LPPs to pictures of spiders than to other emotionally arousing pictures, $F \mathrm{~s}(1,19)<1.6, n s$; whereas in the control group, pictures of spiders elicited significantly smaller LPPs than unpleasant and pleasant pictures, $F_{\mathrm{S}}(1,22)>8.5$, $p$ s $<.01$.
Phobia-Relevant (Spiders)

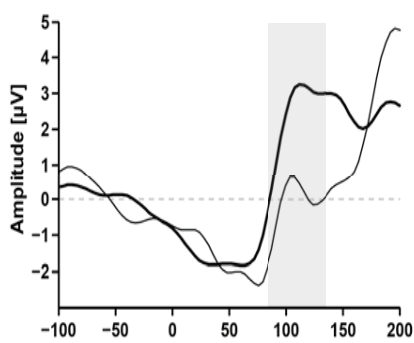

Neutral
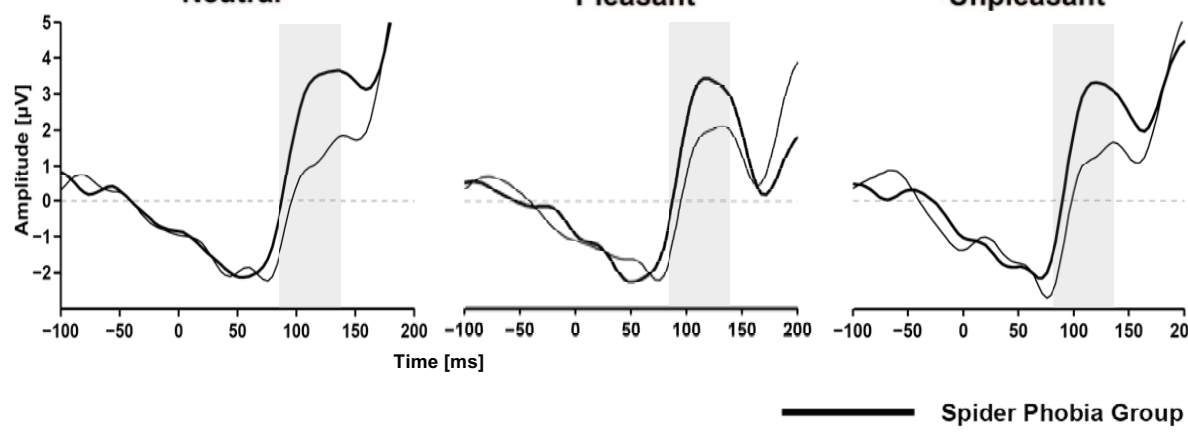

Unpleasant

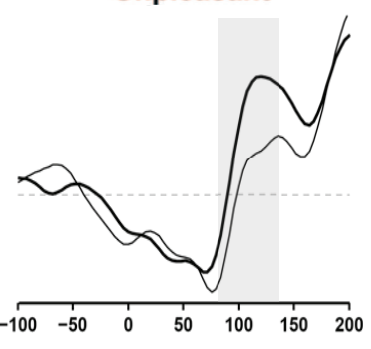

Spider Phobia Group

Control Group

Scalp Difference: Spider Phobia - Control Group
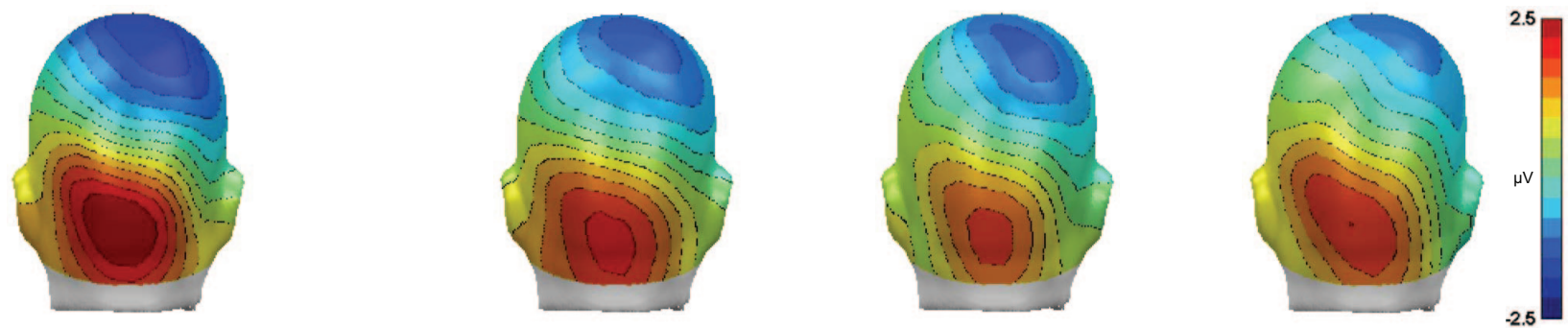

Figure 2. The top graphs illustrate grand-averaged event-related potential waveforms for a representative left occipital sensor (\#71) elicited in individuals with spider phobia and controls by phobia-relevant spider pictures, as well as neutral, pleasant, and unpleasant material. Shaded areas mark the time interval of 80 to $132 \mathrm{~ms}$ selected for the analysis of the P1. The bottom graphs illustrate scalp potential difference maps (spider phobics minus controls) separately for the four picture categories (see top graphs). 
Phobia-Relevant (Spiders)

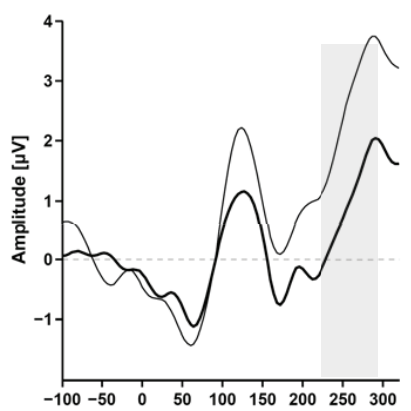

Neutral

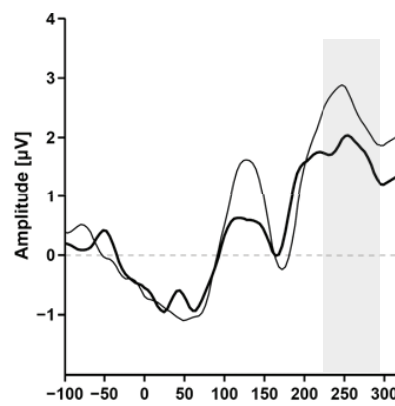

Pleasant

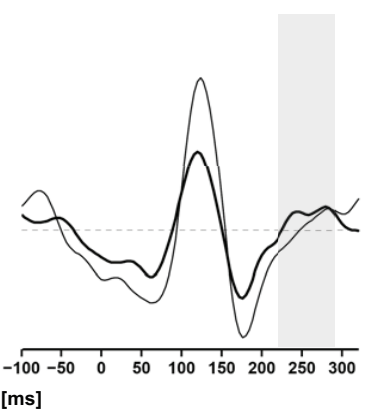

Unpleasant

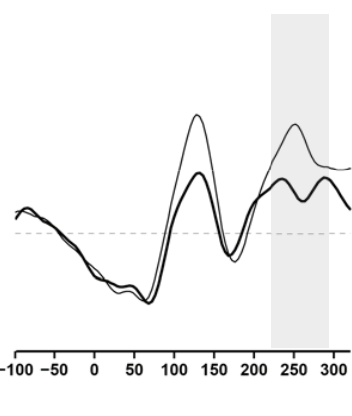

\section{Scalp Difference: Spider Phobia - Control Group}
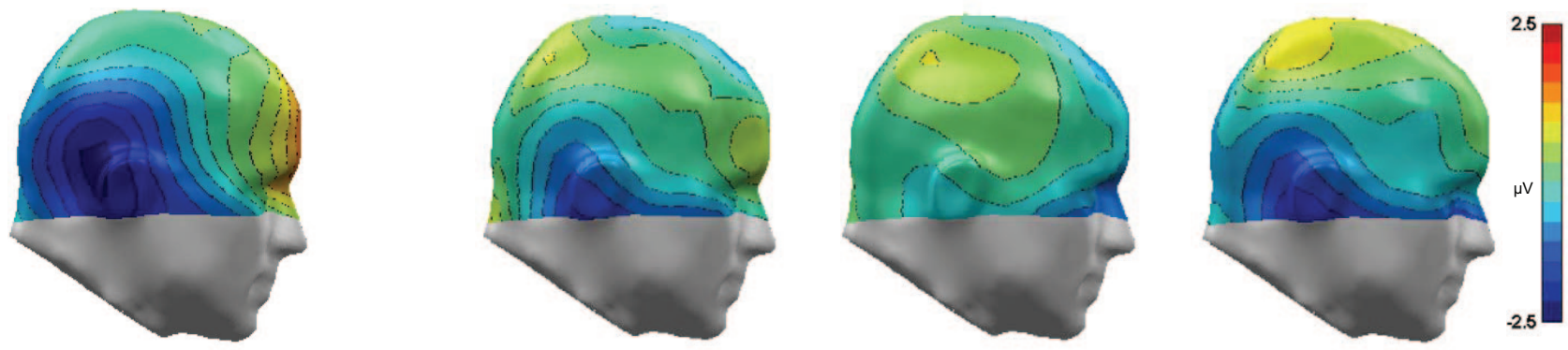

Figure 3. The top graphs illustrate grand-averaged event-related potential waveforms for a representative right temporo-occipital sensor (\#98) elicited in individuals with spider phobia and controls by phobia-relevant spider pictures, as well as neutral, pleasant, and unpleasant material. Shaded areas mark the time interval of 240 to $292 \mathrm{~ms}$ selected for the statistical analysis of the early posterior negativity. The bottom graphs illustrate scalp potential difference maps (spider phobics minus controls) separately for the four picture categories (see top graphs).

\section{Discussion}

The present study investigated the temporal dynamics of visual attention to phobia-relevant, pleasant, and unpleasant cues in spider phobia and nonphobic volunteers. Providing a millisecond time resolution of dynamic brain activity, ERP measures revealed differences in the processing of spider pictures between participants with spider phobia and nonphobic control participants at distinct processing stages. The P1 component was the first ERP component revealing group differences, similarly pronounced for phobia-relevant and control pictures. At later stages of perceptual and evaluative processing indexed by the EPN and LPP, effects of preferential processing appeared specific to the processing of phobia-relevant materials in the spider phobia group. However, this attentional bias to phobia-related stimuli was not larger than that evoked by other highly arousing emotional stimuli. Taken together, in contexts in which phobiarelevant materials are likely, small animal phobics reveal a transition in stimulus perception from unspecific hypervigilance to all stimuli toward the fear-specific enhancement in attention.

\section{Hypervigilance in Spider Phobia Participants at Early Stages of Stimulus Processing}

Relative to nonfearful controls, spider phobia participants responded with larger P1 amplitudes to all visual stimuli irrespective of their emotional contents. Thus, previous findings regarding the processing of schematic presentations of spiders were replicated using naturalistic stimulus presentations (Kolassa et al., 2006). As both groups viewed the same stimuli, these early modulatory effects on the ERPs cannot be explained by differences in physical stimulus characteristics. Instead, these effects are presumed to reflect differences in the attentional state among groups. In light of previous evidences for emotionally related facilitation of the P1 attention effects (Pourtois et al., 2004), these findings may reflect the increased allocation of attentional resources to potentially fear-relevant images. Specifically, a fixation cross was presented in the center of the screen signaling the participants that a visual stimulus would be presented at this location after $500 \mathrm{~ms}$. Thus, the increased P1 amplitudes to phobia-related and control pictures in spider phobia might reflect hypervigilance and increased attention to a location at which fear-related images might occur. These findings may be further considered from the perspective of the predator imminence model proposed by Fanselow (1994) and elaborated by Lang, Bradley, and Cuthbert (1997). According to this model, defensive behavior is organized in several stages depending upon the proximity of the potential threat. During the first stage of the defense cascade (pre-encounter defense), preemptive behavior including threat-unspecific vigilance is engaged (Davis \& Lang, 2003). Accordingly, the fixation cross may engage 

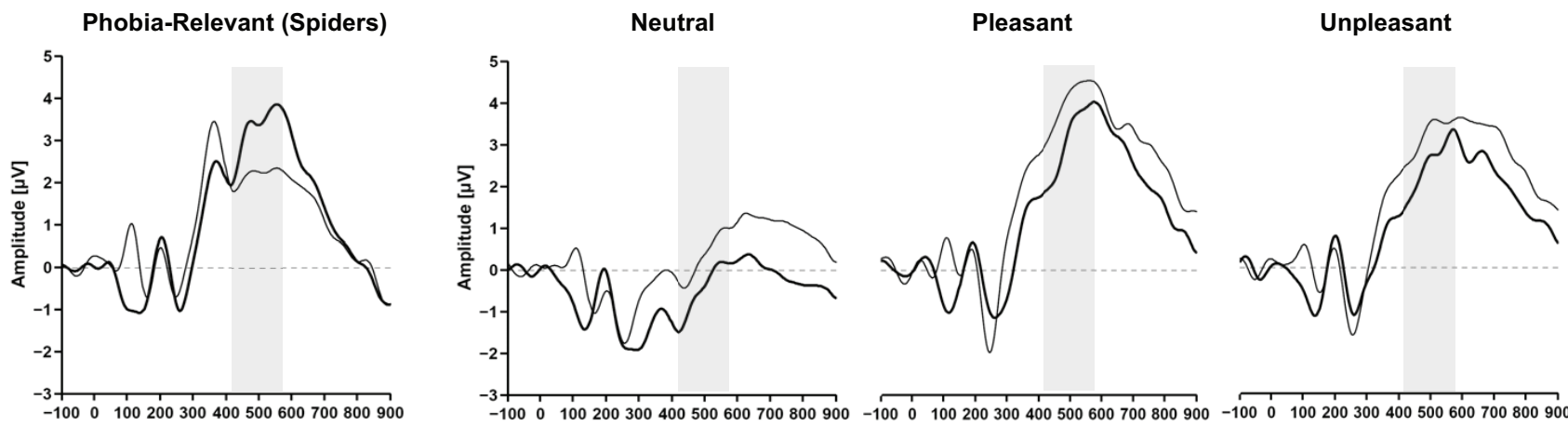

Time $[\mathrm{ms}]$

Spider Phobia Group

Scalp Difference:

Control Group

\section{Spider Phobia - Control Group}
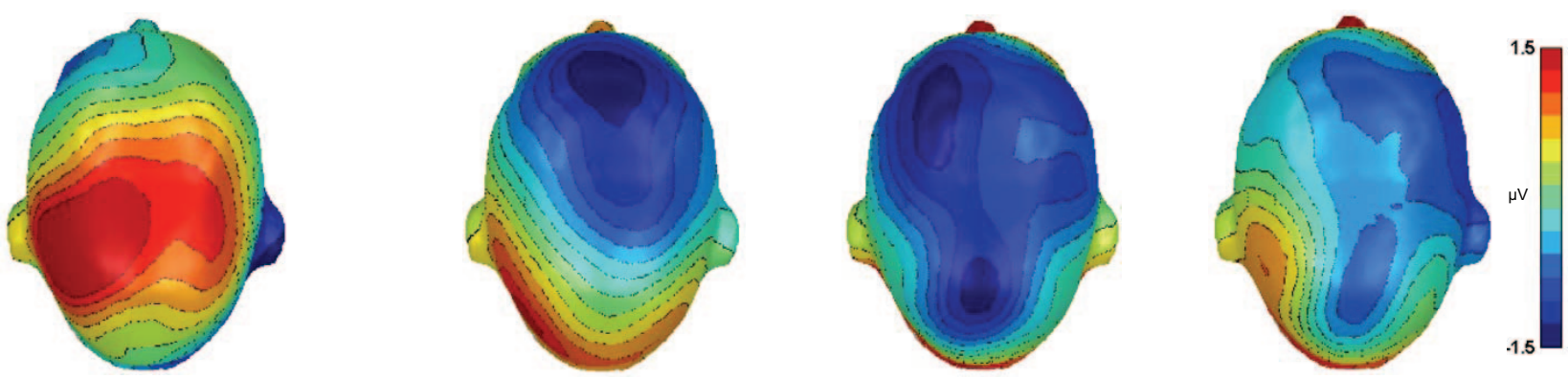

Figure 4. The top graphs illustrate grand-averaged event-related potential waveforms for a representative right centro-parietal sensor (\#88) elicited in individuals with spider phobia and controls by spider pictures, as well as neutral, pleasant, and unpleasant material. Shaded areas mark the time interval of 428 to 588 ms selected for the analysis of the late positive potential. The bottom graphs illustrate scalp potential difference maps (spider phobics minus controls) separately for the four picture categories (see top graphs).

a state of threat-unspecific hypervigilance to all visual stimuli in spider phobic participants, nonspecifically enhancing early perceptual processing reflected by the P1- component. After this initial hypervigilance to all stimuli, individuals with specific phobia shift into a mode of increased attention to emotionally significant and threat-related stimuli once these stimuli are identified and encoded. As a caveat, it has to be noted that we cannot rule out that participants with spider phobia would show increased P1 amplitudes to external stimuli even in a context in which no spider pictures would be expected since such a condition was not tested in the current study.

\section{Preferential Processing of Phobia-Relevant and Other Emotional Stimuli: Early Posterior Negativity}

The present study revealed increased EPN amplitudes to spider pictures in spider phobia compared to the control group over right posterior sensor sites. In contrast to the P1 effect, these group differences were specific for the phobia-relevant materials suggesting a preferential emotional processing for these stimuli in individuals with specific phobia. Although these findings are in line with results from a previous ERP study (Kopp \& Altman, 2005), three further studies did not observe the modulation of ERP components preceding the LPP during the processing of phobiarelevant pictures (Kolassa et al., 2005, 2006; Miltner et al., 2005). Methodological differences may account for inconsistent findings among studies. In the present study, the electrocortical activity was recorded during a passive viewing condition, while in the study by Kolassa et al. (2005) picture viewing was combined with a topdown color and object identification task that might have resulted in a different distribution of attentional resources. A main difference among the present study and a further study of Kolassa et al. (2006) concerns the stimulus materials. Rather than presenting schematic drawings, naturalistic images were shown in the present study. Furthermore, the Miltner et al. study (Miltner et al., 2005) did not record the electrocortical activity from inferior occipitotemporal sensor locations revealing the differential spider picture processing in the present study. Taken together, the present study provides further evidence for the preferential processing of phobiarelated stimulus materials preceding the LPP component. However, this issue needs to be explored in future research providing a systematic comparison of passive picture viewing and explicit attention instructions and relying on a broader range of fear cues.

The present study also replicated previous findings in that pleasant and unpleasant cues elicited enlarged EPN amplitudes 
Late Positive Potentials

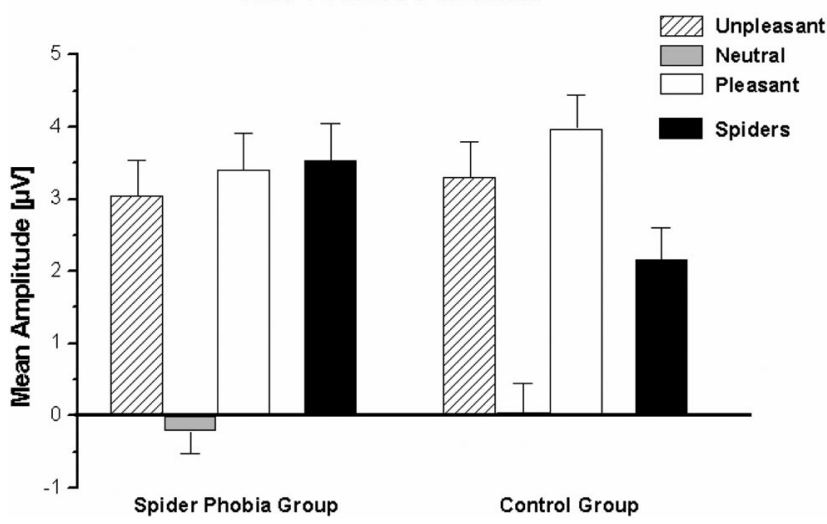

Figure 5. Effect of emotional intensity and phobia relevance: Mean late positive potential amplitudes (and standard errors) for the spider phobia and control group averaged from the centro-parietal sensor cluster in the time interval of 428 to $588 \mathrm{~ms}$ after picture onset.

compared to neutral images. Furthermore, previous research showed that the EPN effect was most apparent for emotional materials judged as high in arousal (Schupp et al., 2003). For individuals with spider phobia, pictures of spiders are emotionally arousing stimuli (Hamm et al., 1997). Accordingly, the enhanced EPN to phobia-related pictures in spider compared to the control group may reflect the increased stimulus salience of fear-related cues. Considered from the perspective of the defense cascade model, these EPN findings indicate that the initial hypervigilance to all stimuli, that is, pre-encounter defense indexed by the unspecific group differences in the $\mathrm{P} 1$ component, is followed by a shift into postencounter defense, prompted by the detection of the threat stimulus. A recent study examining the relation of the P1 and EPN component to stimulus identification and preferential emotion processing is consistent with this reasoning. Varying picture perceptibility by visual noise, the P1 was inversely related to the amount of visual noise. Subsequently, the discrimination among emotional and neutral materials was revealed by the EPN component but only in conditions supporting stimulus identification (Schupp, Stockburger, Schmälzle, Bublatzky, Weike, \& Hamm, 2008). Thus, increased P1 amplitudes may facilitate processes of stimulus identification preceding content-specific modulatory effects contingent on initial stimulus categorization. Overall, the present findings show that fear-specific cues trigger an enhanced EPN amplitude in phobics presumed to reflect the "tagging" for preferential processing in later processing periods (Schupp et al., 2006).

\section{Elaborated Processing of Phobia-Relevant Stimuli: Late Positive Potentials}

Replicating previous findings (Kolassa et al., 2005; Kopp \& Altman, 2005; Miltner et al., 2005), pictures of spiders evoked significantly larger LPPs in participants with specific phobia than in controls. The LPP enhancement was fear specific as control pictures elicited comparable LPP amplitudes in the spider and control group. The enlarged LPP amplitudes to phobia-relevant stimuli in phobics may reflect a state of enhanced attention to feared stimulus materials, which is related to stimulus recognition and elaborated processing of the significant stimulus. From the perspective of the defense model, these data suggest that the attention toward the threatening cue is selectively increased before defensive action is initiated. These data would support the model proposed by Fanselow (1994) suggesting that this switch from an attentional mode (increased LPPs) to an active defense mode (indexed by cardiac acceleration; see Hamm et al., 1997) may involve a switch from activation of the ventral to the dorsal periaqueductal gray. The ventral part projects to brain centers that modulate bradycardia and freezing, and the dorsal part projects to cardiovascular systems that mediate tachycardia and active escape behavior (see Davis \& Lang, 2003 for a detailed discussion).

\section{Attentional Bias to Emotionally Significant Events}

Visual attention can be voluntarily directed toward stimuli and is also attracted by stimuli that are emotionally significant. In the present study, individuals viewed the pictures without any explicit cognitive task. Thus, the increased EPN and LPP to phobia-related stimuli suggest that these stimuli involuntarily attract attention in individuals with specific phobia. These findings could support cognitive theories of anxiety that postulate an attentional bias toward threatening stimuli as a vulnerability factor for developing anxiety disorders (Mathews \& Mackintosh, 1998; Mogg \& Bradley, 1998; Williams et al., 1997). On the other hand, enhanced EPN and LPP were also found for other unpleasant und pleasant arousing stimuli, further supporting the robust evidence from previous research (Schupp et al., 2003, 2006). Moreover, the increase in amplitude both for EPN and LPP were comparable for phobiarelevant and other emotionally significant stimuli within the phobia group. These data are consistent with results from functional imaging studies by Sabatinelli and coworkers (2005) who found increased amygdala and visual cortex activation in snake-phobic participants when they were exposed to pictures of snakes. However, the same increase in activation in both areas was also found during encoding of highly arousing pleasant and unpleasant pictures and did not differ from the activation during viewing of snake pictures in the snake-phobic participants. The same pattern of results was recently observed for individuals with spider phobia in a study by Wendt et al. (2008).

Taken together these data suggest that, although participants with specific phobia show enhanced selective processing of their phobia-relevant cues, the increased attention capture of these cues does not seem to be threat specific but is rather related to the motivational significance of the emotional cues. Thus, the increased attention effects observed in individuals with specific phobia might not be a result of a top-down attentional bias effects as would be suggested by cognitive theories of fear and anxiety. A more probable explanation of these findings might be, that the emotional system involuntarily (i.e., without explicit instruction to do so) drives the cognitive system for elaborated processing of emotional significant cues to facilitate appropriate action (Lang \& Davis, 2006). On the other hand, the current ERP data also show clear evidence for the influence of top-down attention effects. Informing spider fearful participants that a phobia-related cue might occur in the current context puts the organism in the state of general hypervigilance toward all visual stimuli that are presented in such environment irrespective of their emotional content. These data support a dynamic view of the interaction of attention and 
emotion as was put forward in the defense cascade model outlined above. In a context, where a threat cue might occur (preencounter defense or anticipation of threat) the organism shows a general hypervigilance to all stimuli in the environment. Once the threat is detected (postencounter defense), attention is selectively captured by the threat cue. This attention capture, however, is not stronger than that by other emotionally significant cues suggesting that emotion is driving this preferential processing effect. If the threat cue does not disappear or even approaches, defensive response mobilization is engaged, for example, favoring escape as in the case of animal phobia. This phobia-specific response mobilization can be indexed by cue-specific cardiac acceleration and startle potentiation that clearly exceeds startle facilitation during viewing of other unpleasant materials (Hamm et al., 1997).

In summary, the results from the present ERP study confirm previous evidence for preferential sensory processing of fearrelevant cues (Kolassa et al., 2005; Kopp \& Altmann, 2005; Miltner et al., 2005; Öhman et al., 2001) and showed that this fear-related modulation occurred already at early processing stages. Furthermore, our study supports the hypothesis that high fearful subjects exhibit an unspecific hypervigilance in the context associated with threatening objects (Fanselow, 1994) and an early involuntary tagging of fear-relevant stimuli that might assist in selecting them for further elaborated processing. Confirming the data obtained by Sabatinelli et al. (2005), the effects of preferential processing for fear-relevant stimuli appear not to be threat specific but are rather related to the emotional relevance of these cues. Accordingly, our data do not support the assumptions formulated in cognitive theories of anxiety that postulate an attentional bias toward threatening stimuli as a vulnerability factor for developing anxiety disorders (Mathews \& Mackintosh, 1998; Mogg \& Bradley, 1998; Williams, Watts, MacLeod, \& Mathews, 1997) and rather suggest that the enhanced attention toward feared objects can be driven by the emotional system that learned to overestimate their emotional value to facilitate appropriate action (Lang \& Davis, 2006). Future studies need to determine the generality of our findings and explore the increased attention effects in various levels of anxious apprehension and task demands.

\section{References}

Bradley, M. M., \& Lang, P. J. (1994). Measuring emotion: The SelfAssessment Manikin and the semantic differential. Journal of Behavior Therapy and Experimental Psychiatry, 25, 49-59.

Carlsson, K., Petersson, K. M., Lundquist, D., Karlsson, A., Ingvar, M., \& Öhman, A. (2004). Fear and the amygdala: Manipulation of awareness generates differential cerebral responses to phobic and fear-relevant (but nonfeared) stimuli. Emotion, 4, 340-353.

Clark, V. P., \& Hillyard, S. A. (1996). Spatial selective attention affects early extrastriate but not striate components of the visual evoked potential. Journal of Cognitive Neuroscience, 8, 387-402.

Curtis, G. C., Magee, W. J., Eaton, W. W., Wittchen, H-U., \& Kessler, R. C. (1998). Specific fears and phobias: Epidemiology and classification. British Journal of Psychiatry, 173, 212-217.

Davis, M., \& Lang, P. J. (2003). Emotion. In M. Gallagher \& R. J. Nelson (Eds.), Handbook of psychology: Biological psychology (Vol. 3, pp. 405-439). Hoboken, NJ: Wiley.

Del Cul, A., Baillet, S., \& Dehaene, S. (2007). Brain dynamics underlying the nonlinear threshold for access to consciousness. PloS Biology, 5, e260 doi :10.1371/journal.pbio.0050260.

Dilger, S., Straube, T., Mentzel, H. J., Fitzek, C., Reichenbach, H. R.,
Hecht, H., et al. (2003). Brain activation to phobia-related pictures in spider phobic humans: An event-related functional magnetic resonance imaging study. Neuroscience Letters, 348, 29-32.

Fanselow, M. S. (1994). Neural organization of the defensive behavior system responsible for fear. Psychonomic Bulletin and Review, 1, 429438.

Fredrikson, M. (1981). Orienting and defensive reactions to phobic and conditioned fear stimuli in phobics and normals. Psychophysiology, 18, 456-465.

Fredrikson, M., \& Furmark, T. (2003). Amygdaloid regional cerebral blood flow and subjective fear during symptom provocation in anxiety disorders. Annals of the New York Academy of Sciences, 985, 341-347.

Fredrikson, M., Wik, G., Annas, P., Ericson, K., \& Stone-Erlander, S. (1995). Functional neuroanatomy of visually elicited simple phobic fear: Additional data and theoretical analysis. Psychophysiology, 32, 43-48.

Fredrikson, M., Wik, G., Greitz, T., Eriksson, K., Ericsson, C., StoneErlander, S., et al. (1993). Regional cerebral blood flow during experimental phobic fear. Psychophysiology, 30, 127-131.

Globisch, J., Hamm, A., Esteves, F., \& Öhman, A. (1999). Fear appears fast: Temporal course of startle reflex potentiation in animal fearful subjects. Psychophysiology, 36, 66-75.

Hamm, A., Cuthbert, B. N., Globisch, J., \& Vaitl, D. (1997). Fear and the startle reflex: Blink modulation and autonomic response patterns in animal and mutilation fearful subjects. Psychophysiology, 34, 97-107.

Hamm, A. O. (2006). Spezifische Phobien. Göttingen: Hogrefe.

Hamm, A. O., Schupp, H. T., \& Weike, A. I., (2003). Motivational organization of emotions: Autonomic changes, cortical responses, and reflex modulation. In R. J. Davidson, K. R. Scherer, \& H. Hill Goldsmith (Eds.), Handbook of affective sciences (pp. 187-211). New York: Oxford University Press.

Hare, R. D. (1973). Orienting and defensive responses to visual stimuli. Psychophysiology, 10, 453-464.

Junghöfer, M., Elbert, T., Tucker, D., \& Rockstroh, B. (2000). Statistical control of artifacts in dense array EEG/MEG studies. Psychophysiology, $37,523-532$.

Kolassa, I. T., Musial, F., Kolassa, S., \& Miltner, W. H. R. (2006) Event-related potentials when identifying or color-naming threatening schematic stimuli in spider phobic and non-phobic individuals. $B M C$ Psychiatry, 6, 38.

Kolassa, I. T., Musial, F., Mohr, A., Trippe, R. H., \& Miltner, W. H. R. (2005). Electrophysiological correlates of threat processing in spider phobics. Psychophysiology, 42, 520-530.

Kopp, B., \& Altmann, R. (2005). Neurocognitive effects of phobia-related stimuli in animal-fearful individuals. Cognitive, Affective, \& Behavioral Neuroscience, 5, 373-387.

Lang, P. J., Bradley, M. M., \& Cuthbert, B. N. (1997). Motivated attention: Affect, activation and action. In P. J. Lang, R. F. Simons, \& M. T. Balaban (Eds.), Attention and orienting: Sensory and motivational processes (pp. 97-135). Hillsdale, NJ: Erlbaum, Inc.

Lang, P. J., Bradley, M. M., \& Cuthbert, B. N. (2005). International affective picture system (IAPS): Digitized photographs, instruction manual and affective ratings. (Tech. Rep. No. A-6). Gainesville, FL: University of Florida.

Lang, P. J., \& Davis, M. (2006). Emotion, motivation, and the brain: Reflex foundations in animal and human research. Progress in Brain Research, $156,3-29$

Lang, P. J., Davis, M., \& Öhman, A. (2000). Fear and anxiety: Animal models and human cognitive psychophysiology. Journal of Affective Disorders, 61, 137-159.

LeDoux, J. E. (1987). Emotion. In F. Plum \& V. B. Mountcastle (Eds.), Handbook of physiology: Nervous system V, higher function (pp. 419460). Washington, DC: American Physiological Society.

Mangun, G. R., Hillyard, S. A., \& Luck, S. J. (1993). Electrocortical substrates of visual selective attention. In D. Meyer \& S. Kornblum 
(Eds.), Attention and performance XIV (pp. 219-243). Cambridge, MA: MIT Press.

Mathews, A., \& Mackintosh, B. (1998). A cognitive model of selective processing in anxiety. Cognitive Therapy and Research, 22, 539-560.

Miltner, W. H. R., Trippe, R. H., Krieschel, S., Gutberlet, I., Hecht, H., \& Weiss, T. (2005). Event-related brain potentials and affective responses to threat in spider/snake-phobic and non-phobic subjects. International Journal of Psychophysiology, 57, 43-52.

Mogg, K., \& Bradley, B. (1998). A cognitive-motivational analysis of anxiety. Behaviour Research and Therapy, 36, 809-848.

Nieuwenhuis, S., Aston-Jones, G., \& Cohen, J. D. (2005). Decision making, the P3, and the locus coeruleus-norepinephrine system. Psychological Bulletin, 131, 510-532.

Öhman, A., Flynkt, A., \& Esteves, F. (2001). Emotion drives attention: Detecting the snake in the grass. Journal of Experimental Psychology: General, 130, 466-478.

Öhman, A., \& Mineka, S. (2001). Fears, phobias, and preparedness: Toward an evolved module of fear and fear learning. Psychological Review, 108, 483-522.

Paquette, V., Lévesque, J., Mensour, B., Leroux, J-M., Beaudoin, G., Bourgouin, P., et al. (2003). Change the mind and you change the brain: Effects of cognitive-behavioral therapy on the neural correlates of spider phobia. NeuroImage, 18, 401-409.

Pourtois, G., Grandjean, D., Sander, D., \& Vuilleumier, P. (2004). Electrophysiological correlates of rapid spatial orienting towards fearful faces. Cerebral Cortex, 14, 619-633.

Prigatano, G. P., \& Johnson, H. J. (1974). Autonomic nervous system changes associated with a spider phobic reaction. Journal of Abnormal Psychology, 83, 169-177.

Sabatinelli, D., Bradley, M. M., Fitzsimmons, J. R., \& Lang, P. J. (2005).
Parallel amygdala and inferotemporal activation reflect emotional intensity and fear relevance. Neurolmage, 24, 1265-1270.

Schienle, A., Schäfer, A., Walter, B., Stark, R., \& Vaitl, D. (2005). Elevated disgust sensitivity in blood phobia. Cognition \& Emotion, 19, $1229-1241$.

Schupp, H. T., Flaisch, T., Stockburger, J., \& Junghöfer, M. (2006) Emotion and attention: Event-related brain potential studies. Progress in Brain Research, 156, 31-51.

Schupp, H. T., Junghöfer, M., Weike, A. I., \& Hamm, A. O. (2003). Attention and emotion: An ERP analysis of facilitated emotional stimulus processing. Neuroreport, 14, 1107-1110.

Schupp, H. T., Stockburger, J., Schmälzle, R., Bublatzky, F., Weike, A. I., \& Hamm, A. O. (2008). Visual noise effects on emotion perception: Brain potentials and stimulus identification. Neuroreport, 19, 167-171.

Sergent, C., Baillet, S., \& Dehaene, S. (2005). Timing of the brain events underlying access to consciousness during the attentional blink. Nature Neuroscience, 8, 1391-1400.

Straube, T., Mentzel, H. J., \& Miltner, W. H. R. (2006). Neural mechanisms of automatic and direct processing of phobogenic stimuli in specific phobia. Biological Psychiatry, 59, 162-170.

Wendt, J., Lotze, M., Weike, A. I., Hosten, N., \& Hamm, A. O. (2008) Brain activation and defensive response mobilization during sustained exposure to phobia-related and other affective pictures in spider phobia. Psychophysiology, 45, 205-215.

Williams, J. M. G., Watts, F. N., MacLeod, C., \& Mathews, A. (1997). Cognitive psychology and emotional disorders. Chichester, UK: Wiley. 